\title{
Metamaterial-Based Compact Filter Design
}

\author{
Merih Palandöken \\ Berlin Institute of Technology \\ Germany
}

\section{Introduction}

Emerging requirements of increasingly complex wireless systems necessitate novel design methods of wireless components to be developed for the fulfillment of many performance criteria simultaneously. One of these wireless components to be enhanced for high data rate transmission systems and matched with the new challenges in new generation communication systems is the microwave filter. This chapter is mainly dealing with the novel microwave filter design methods based on artificial materials. How to design electrically small resonators and to couple each of these resonators with the successive resonators in a periodic/aperiodic manner in addition to the feeding line for the bandpass and bandstop filter designs are highlighted throughout this chapter.

In this chapter, because the current trend in filter design is the filter miniaturization for more compact wireless systems, basic approaches in electrically small filter design based on artificial metamaterials are explained. The compact resonators result the signal suppression level of the incoming signal in bandstop filter designs and signal selectivity of the transmitted signal in bandpass filter designs to be higher as a performance enhancement. It is due to the target geometrical compactness, resulting into the possibility of cascading more electrically small resonator cells in a restricted area. Because these performance parameters are highly related with $\mathrm{Q}$ factor of each individual resonator, the eigenmodes of periodically loaded negative permittivity and negative permeability resonators are analytically calculated in Section 2. The passband frequencies and $Q$ factor of the filter to be designed can be engineered with the geometrical and topological parameters of the resonators, which is the fundamental idea of artificial material based filter designs. This analytical calculation points out the effect of electromagnetic material parameters of negative permittivity and permeability materials on filter performance. In Section 3 and Section 4, two compact filter designs are proposed and explained along with the numerical results as metamaterial based filter examples. One of these designs is the fractal resonator based bandstop filter. The other design is the compact bandpass filter based on thin wire loaded spiral resonator cells. These are quite good examples of compact filter designs with negative permeability and left-handed metamaterial unit cells, respectively. These numerically calculated filters points out the compactness and performance enhancement of novel designs in comparison to the conventional filter designs in a restricted volume. 


\section{Theoretical analysis}

The motivating principle of theoretical research on LHM is first introduced by Veselago with his theoretical paper in 1968 [Veselago, 1968]. He considered electromagnetic wave propagation through a homogenous isotropic electromagnetic material in which both permittivity and permeability were assumed to have negative real values. Because the direction of the Poynting vector of a monochromatic plane wave is opposite to that of its phase velocity in such a material, he referred to this medium as left-handed medium. This material property makes in turn these materials to support backward-wave propagation due to its negative refractive index. The natural inexistence of such exotic materials had unfortunately led the motivating ideas of Veselago on negative refraction, its various electromagnetic and optical consequences to receive little attention in the scientific community [Engheta \& Ziolkowski, 2006; Caloz \& Itoh, 2005; Eleftheriades \& Balmain, 2005]. However, the work of Pendry on the electromagnetic engineering of magnetic permeability and electric permittivity of the materials with electrically small metallic inclusions has made Veselago's ideas realisable [Pendry et al.,1999; Pendry et al., 1996]. In 2000, Smith inspired by the work of Pendry and constructed a composite LH medium in the microwave regime by arranging the periodic arrays of small metallic wires and SRRs [Smith \& Kroll, 2000]. He demonstrated the anomalous refraction at the interface of this LH medium with the air, which is the result of negative refraction in this artificial material. The effective electromagnetic parameters were also retrieved experimentally and numerically from the transmission and reflection data to prove the negative refractive index [Smith et al., 2005 ; Alexopoulos et al., 2007; Chen et al., 2004 ; Smith et al., 2000]. There have now been several theoretical and experimental studies that have been reported confirming negative refractive index. There are some engineering applications derived from this concept such as phase compensation and electrically small resonators [Engheta, 2002], negative angles of refraction [Kong et al., 2002; Kolinko \& Smith, 2003; Ziolkowski, 2003], sub-wavelength waveguides with lateral dimensions below diffraction limits [Alu \& Engheta, 2003,2004], enhanced focusing [Grbic \& Eleftheriades,2004], backward wave antennas [Grbic \& Eleftheriades,2002; Caloz \& Itoh, 2005 ], enhanced electrically small antennas [Ziolkowski \& Kipple, 2003 ] and compact microwave filters [Marques et al., 2008].

The main principle of negative refractive index in LHMs can be deduced quite easily by the calculation of TE and TM wave impedances of waveguide modes. The TE $\mathrm{mn}$ and $\mathrm{TM}_{\mathrm{mn}}$ wave impedances are calculated in a general form as

$$
\begin{aligned}
& Z_{T E m n}=\frac{\omega \mu}{k_{z m n}}=-\frac{\omega\left|\mu_{L H M}\right|}{k_{z m n}} \\
& Z_{T M m n}=\frac{k_{z m n}}{\omega \varepsilon}=-\frac{k_{z m n}}{\omega\left|\varepsilon_{L H M}\right|}
\end{aligned}
$$

with the longitudinal wave number, $k_{z m n}$ at the operation frequency of $\omega$ with the magnetic permeability, $\mu$ and electric permittivity, $\varepsilon$ of the waveguide filling material. Because the wave impedance of a passive microwave component is always positive, the phase constant has to be correspondingly negative in the impedance formulation due to the negative permittivity and permeability. As a result, the effective refractive index is also negative. This is an alternative explanation to understand the underlying reasoning why the refractive 
index has to be negative in the artificial materials with negative permittivity and permeability. Due to the exploitation of electrically small resonators in the resonator design, compact resonators can be cascaded to increase the frequency selectivity and stop-band rejection level in a restricted volume. The accompanying degradation of $Q$ factor due to the periodic arrangement of intercoupled cells can be compensated by the implementation of complementary resonators, in which virtual magnetic currents are the excited resonant sources instead of electrical currents flowing through the lossy metal [Marques et al., 2008].

In this chapter, the subwavelength resonance feature of periodically arranged negative permittivity and permeability materials in a rectangular waveguide is explained by the calculation of eigenmode equation for TE modes. The eigenmode calculation can be similarly done for TM modes by replacing TE wave impedance with TM wave impedance in ABCD matrix formulation. The periodically loaded waveguide model is shown in Fig. 1.
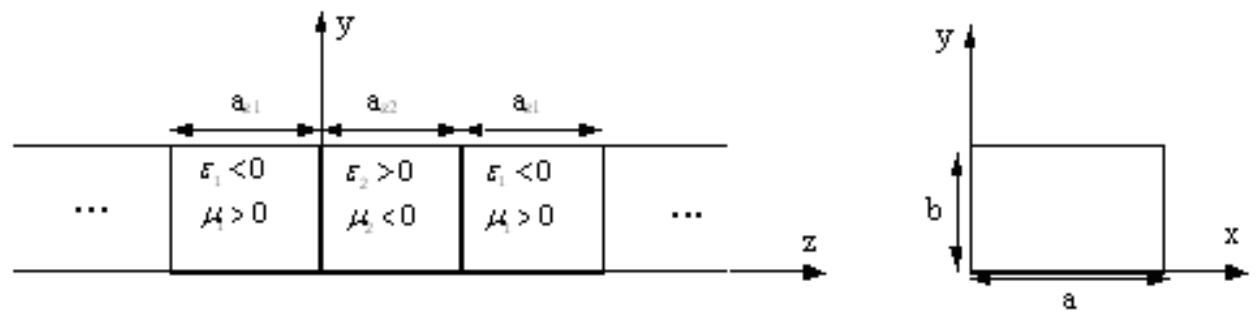

Fig. 1. Waveguide model of periodically arranged negative permeability and permittivity materials

$A B C D$ matrix of negative permittivity material of length $a_{z 1}$ is formulated with TE-wave impedance, $\mathrm{Z}_{\mathrm{H} 1}$ and phase constant, $\mathrm{k}_{\mathrm{z} 1}$ as

$$
\begin{gathered}
M_{A B C D_{-} \text {negeps }}=\left[\begin{array}{cc}
\cos \left(k_{z 1} a_{z 1}\right) & j Z_{H 1} \sin \left(k_{z 1} a_{z 1}\right) \\
j \frac{\sin \left(k_{z 1} a_{z 1}\right)}{Z_{H 1}} & \cos \left(k_{z 1} a_{z 1}\right)
\end{array}\right] \\
k_{z 1}=\sqrt{\omega^{2} \mu_{1} \varepsilon_{1}-\left(\frac{m \pi}{a}\right)^{2}-\left(\frac{n \pi}{b}\right)^{2}} \\
Z_{H 1}=\frac{\omega \mu_{1}}{k_{z 1}}
\end{gathered}
$$

where $\mathrm{a}$ and $\mathrm{b}$ are the waveguide side lengths in $\mathrm{x}$ and $\mathrm{y}$ directions, respectively.

In the same manner, $A B C D$ matrix of negative permeability material of length $a_{\mathrm{z} 2}$ is formulated with TE-wave impedance, $\mathrm{Z}_{\mathrm{H} 2}$ and phase constant, $\mathrm{k}_{\mathrm{z} 2}$ as

$$
M_{A B C D_{-} \text {negmu }}=\left[\begin{array}{cc}
\cos \left(k_{z 2} a_{z 2}\right) & j Z_{H 2} \sin \left(k_{z 2} a_{z 2}\right) \\
j \frac{\sin \left(k_{z 2} a_{z 2}\right)}{Z_{H 2}} & \cos \left(k_{z 2} a_{z 2}\right)
\end{array}\right]
$$




$$
\begin{gathered}
k_{z 2}=\sqrt{\omega^{2} \mu_{2} \varepsilon_{2}-\left(\frac{m \pi}{a}\right)^{2}-\left(\frac{n \pi}{b}\right)^{2}} \\
Z_{H 2}=\frac{\omega \mu_{2}}{k_{z 2}}
\end{gathered}
$$

Thus, ABCD matrix of one unit cell of length, $\mathrm{a}_{\mathrm{z} 1}+\mathrm{a}_{\mathrm{z} 2}$, consisting of the negative permeability and permittivity materials is calculated by cascading $A B C D$ matrices of the respective materials as

$$
\begin{gathered}
M_{A B C D_{-} \text {cell }}=\left[\begin{array}{cc}
\cos \left(k_{z 2} \frac{a_{z 2}}{2}\right) & j Z_{H 2} \sin \left(k_{z 2} \frac{a_{z 2}}{2}\right) \\
\sin \left(k_{z 2} \frac{a_{z 2}}{2}\right) & \cos \left(k_{z 2} \frac{a_{z 2}}{2}\right)
\end{array}\right]\left[\begin{array}{cc}
\cos \left(k_{z 1} a_{z 1}\right) & j Z_{H 1} \sin \left(k_{z 1} a_{z 1}\right) \\
Z_{H 2} & \sin \left(k_{z 1} a_{z 1}\right) \\
Z_{H 1} & \cos \left(k_{z 1} a_{z 1}\right)
\end{array}\right] \\
{\left[\begin{array}{cc}
\cos \left(k_{z 2} \frac{a_{z 2}}{2}\right) & j Z_{H 2} \sin \left(k_{z 2} \frac{a_{z 2}}{2}\right) \\
\frac{\sin \left(k_{z 2} \frac{a_{z 2}}{2}\right)}{Z_{H 2}} & \cos \left(k_{z 2} \frac{a_{z 2}}{2}\right)
\end{array}\right]} \\
M_{A B D_{-} \text {cell }}=\left[\begin{array}{cc}
A & B \\
C & D
\end{array}\right]
\end{gathered}
$$

where the matrix elements are calculated as in (2.d).

$$
\begin{aligned}
& A=\cos \left(k_{z 1} a_{z 1}\right) \cos \left(k_{z 2} a_{z 2}\right)-\frac{1}{2}\left(\frac{Z_{H 2}}{Z_{H 1}}+\frac{Z_{H 1}}{Z_{H 2}}\right) \sin \left(k_{z 1} a_{z 1}\right) \sin \left(k_{z 2} a_{z 2}\right) \\
& B=j Z_{H 2} \cos \left(k_{z 1} a_{z 1}\right) \sin \left(k_{z 2} a_{z 2}\right)+j \frac{\sin \left(k_{z 1} a_{z 1}\right)}{2 Z_{H 1}}\left(\left(Z_{H 1}^{2}-Z_{H 2}^{2}\right)+\left(Z_{H 1}^{2}+Z_{H 2}^{2}\right) \cos \left(k_{z 2} a_{z 2}\right)\right) \\
& C=j \frac{1}{Z_{H 2}} \cos \left(k_{z 1} a_{z 1}\right) \sin \left(k_{z 2} a_{z 2}\right)+j \frac{\sin \left(k_{z 1} a_{z 1}\right)}{2 Z_{H 1} Z_{H 2}^{2}}\left(\left(-Z_{H 1}^{2}+Z_{H 2}^{2}\right)+\left(Z_{H 2}^{2}+Z_{H 1}^{2}\right) \cos \left(k_{z 2} a_{z 2}\right)\right) \\
& D=\cos \left(k_{z 1} a_{z 1}\right) \cos \left(k_{z 2} a_{z 2}\right)-\frac{1}{2}\left(\frac{Z_{H 2}}{Z_{H 1}}+\frac{Z_{H 1}}{Z_{H 2}}\right) \sin \left(k_{z 1} a_{z 1}\right) \sin \left(k_{z 2} a_{z 2}\right)
\end{aligned}
$$

Thus, the dispersion relation of the periodic array of negative permeability and permittivity materials is calculated from $\mathrm{ABCD}$ parameters to determine the complex propagation constant, $\gamma_{\text {zeff }}$ as

$$
\cosh \left(\gamma_{z e f f}\left(a_{z 1}+a_{z 2}\right)\right)=\cos \left(k_{z 1} a_{z 1}\right) \cos \left(k_{z 2} a_{z 2}\right)-\frac{1}{2}\left(\frac{Z_{H 1}}{Z_{H 2}}+\frac{Z_{H 2}}{Z_{H 1}}\right) \sin \left(k_{z 1} a_{z 1}\right) \sin \left(k_{z 2} a_{z 2}\right)
$$

This dispersion relation can be formulated in an alternative form for the case of ideal lossless material parameters as 


$$
\cosh \left(\gamma_{z e f f}\left(a_{z 1}+a_{z 2}\right)\right)=\cosh \left(\alpha_{z 1} a_{z 1}\right) \cosh \left(\alpha_{z 2} a_{z 2}\right)-\frac{1}{2}\left(\frac{Z_{1}}{Z_{2}}+\frac{Z_{2}}{Z_{1}}\right) \sinh \left(\alpha_{z 1} a_{z 1}\right) \sinh \left(\alpha_{z 2} a_{z 2}\right)
$$

with the wave reactances, $Z_{1,2}$ and attenuation constants, $\alpha_{z 1,2}$ of the negative permittivity and permeability media, respectively. The wave reactances and attenuation constants are calculated from (2.a) and (2.b) in the form of

$$
\begin{gathered}
Z_{H 1}=\frac{-j \sqrt{\omega^{2} \mu_{1}\left|\varepsilon_{1}\right|+\left(\frac{m \pi}{a}\right)^{2}+\left(\frac{n \pi}{b}\right)^{2}}}{Z_{H 2}=}=j Z_{1} \\
j \sqrt{\omega^{2}\left|\mu_{2}\right| \varepsilon_{2}+\left(\frac{m \pi}{a}\right)^{2}+\left(\frac{n \pi}{b}\right)^{2}}=-j Z_{2} \\
\alpha_{z 1}=\sqrt{\omega^{2}\left|\varepsilon_{1}\right| \mu_{1}+\left(\frac{m \pi}{a}\right)^{2}+\left(\frac{n \pi}{b}\right)^{2}} \\
\alpha_{z 2}=\sqrt{\omega^{2} \varepsilon_{2}\left|\mu_{2}\right|+\left(\frac{m \pi}{a}\right)^{2}+\left(\frac{n \pi}{b}\right)^{2}}
\end{gathered}
$$

As it is deduced from (3.b), the wave impedances of negative permittivity and permeability materials are inductive and capacitive, respectively. Thus, the wave propagation can be in principle obtained at any frequency by engineering the material parameters and adjusting the slab thicknesses correspondingly to satisfy the resonance condition. Because of the parametric dependence of eigenmode equation on monotonically increasing functions, only one resonance frequency is calculated for a certain phase shift per cell. This results only one frequency band to be obtained in the dispersion diagram for each transversal wave number in the case of lossless material parameters with low material dispersion. This property makes the novel cavity resonators with one resonance frequency for each transversal wave number to be designed by loading the negative permittivity material with the negative permeability material. However, this is not possible in the resonator designs by longitudinal pairing of two RHMs or one RHM with LHM [Engheta \& Ziolkowski, 2006].

Another potential application is the design of monomode waveguides with arbitrary thick lateral dimension by loading the waveguide walls with the negative permeability and permittivity materials laterally instead of longitudinally [Alu \& Engheta, 2003]. Another important issue is the design of a cavity resonator with negative material parameters at a predetermined resonance frequency, which can be derived from the eigenmode equation. Eigenmode equation indicates that the resonance frequency of a cavity resonator is only dependent on the ratio of each slab thickness rather than the total slab thickness unlike in the conventional resonators [Engheta, 2002]. The same result can also be concluded for the transmission line and resonator designs, which are based on pairing of LH and RH materials in the same unit cell but not of two RH materials. This property leads the compact subwavelength transmission media and resonators to be designed by pairing any slab thicknesses of negative permittivity and permability materials.This is also the main principle in the design of subwavelength guided wave structures with the lateral dimensions below the diffraction limits [Grbic \& Eleftheriades,2004]. 
As a case study, one negative permittivity material of Drude type electric response and one negative permeability material of Lorentzian type magnetic response are periodically arranged inside a rectangular waveguide. The slab lengths of negative permittivity and permeability materials are $1 \mathrm{~mm}$ and $2 \mathrm{~mm}$, respectively. The magnetic resonance, magnetic plasma frequency and loss parameter are $2.10 \mathrm{GHz}, 2.21 \mathrm{GHz}$ and $100 \mathrm{~Hz}$ for the negative permeability material. The electric plasma frequency and loss parameter are $10 \mathrm{GHz}$ and 100 $\mathrm{Hz}$ for the negative permittivity material. As deduced from the material parameters, the material losses are taken into account in the model, however, kept quite small to have low transmission loss and observe the transmission band broadening. The dispersion diagram and Bloch impedance are analytically calculated and shown in Fig. 2 between the magnetic resonance and plasma frequencies of the negative permeability medium.

As it is deduced from Fig. 2a, the propagation constant is negative between $2.106 \mathrm{GHz}$ and $2.146 \mathrm{GHz}$ with $40 \mathrm{MHz}$ bandwidth. It results approximately into $\mathrm{Q}$ factor of 53 in $\mathrm{LH}$ passband. However, in addition to this LH band, RH band is also obtained between 2.173 $\mathrm{GHz}$ and $2.21 \mathrm{GHz}$ with $37 \mathrm{MHz}$ bandwidth. This $\mathrm{RH}$ passband has an approximate $\mathrm{Q}$ factor of 60 with the center frequency of $2.19 \mathrm{GHz}$. There is $27 \mathrm{MHz}$ bandgap between $\mathrm{LH}$ and $\mathrm{RH}$ bands, extending from $2.146 \mathrm{GHz}$ to $2.173 \mathrm{GHz}$ with the highest signal rejection level at the stop band frequency of $2.16 \mathrm{GHz}$. The emergence of these two bands is mainly related with the included material dispersion and loss. As shown in Fig. 2b, Bloch impedance is high ohmic at the lower edge and low ohmic at the higher edge with no reactive part. Thus, the composite material can be modeled as a combination of parallel and series resonant circuits with the resonance frequencies of $2.146 \mathrm{GHz}$ and $2.173 \mathrm{GHz}$, respectively. In order to confirm this issue and the emergence of $\mathrm{RH}$ band at the higher frequencies, the equivalent circuit model of negative permittivity and permeability materials are illustrated in Fig. 3 [Engheta \& Ziolkowski, 2006].

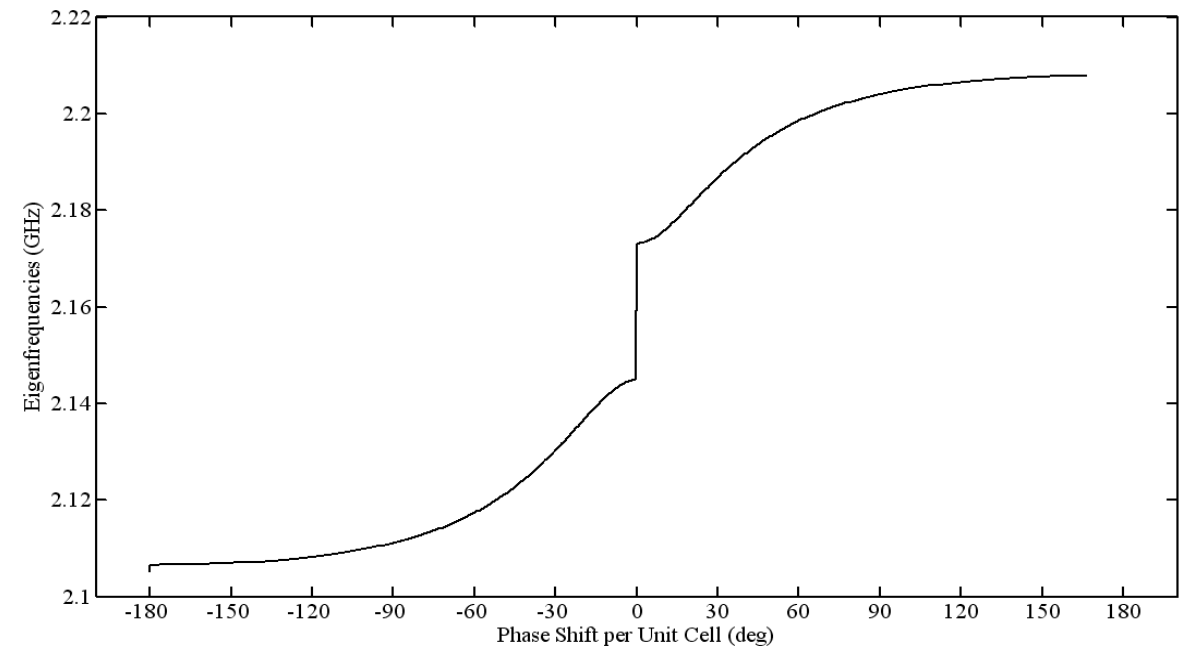

Fig. 2a. 1D dispersion diagram of periodically arranged negative permeability and permittivity materials 


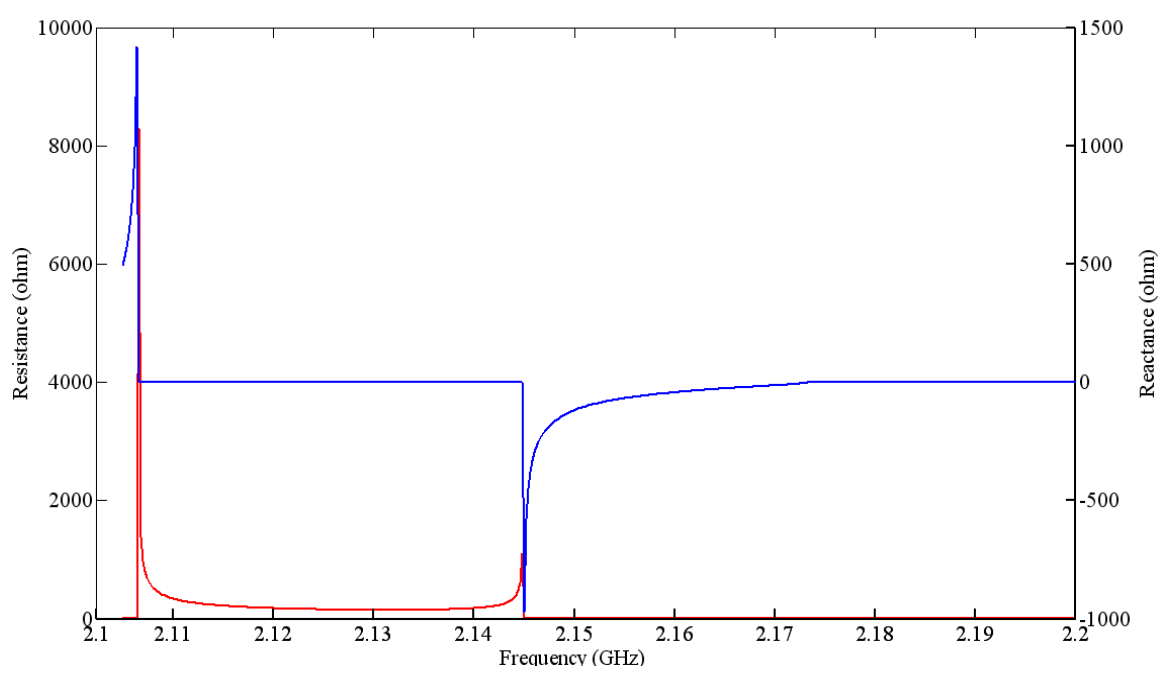

Fig. 2b. Resistance (red) and reactance (blue) of periodically arranged negative permeability and permittivity materials

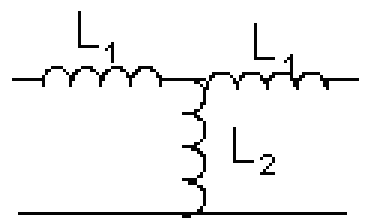

(a)

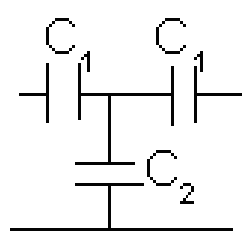

(b)

Fig. 3. Equivalent circuit model of one unit cell of (a) negative permittivity and (b) negative permeability materials

The periodic pairing of negative permittivity material with the negative permeability material results one unit cell to be modeled as

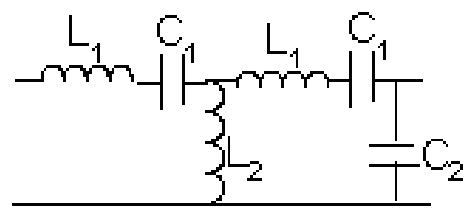

Fig. 4. Equivalent circuit model of one unit cell of periodically loaded negative permittivity and permeability materials

As deduced from Fig.4, for the frequencies larger than the series resonance frequency, the impedance of series branches is inductive. In addition, the resulting impedance of parallel branches are capacitive for the frequencies larger than the parallel resonance frequency. Thus, this circuit model has an equivalent form of series inductor loaded with the shunt capacitor as conventional $\mathrm{RH}$ transmission lines at the frequencies larger than the parallel 
and series resonance frequencies. This is the main reason why this composite material has an additional $\mathrm{RH}$ band at the frequencies larger than the LH band. In a similar manner, the equivalent circuit model of LH materials can be derived by the investigation of the same circuit model for the frequencies smaller than the parallel and series resonance frequencies. For this case, the circuit model has an equivalent form of series capacitor loaded with the shunt inductor. It is the dual of RH circuit model. One important conclusion from these circuit models is that the negative permittivity and permeability materials can be alternatively designed without relying on high lossy resonance phenomenon. In other words, rather than embedding the electrically small resonant metallic inclusions into the host material, the left handed feature can also be realized with low loss by periodic loading of conventional microstrip transmission lines with series capacitors and shunt inductors in planar microwave technology [Caloz \& Itoh, 2005; Eleftheriades \& Balmain, 2005]. Many microwave circuits have been implemented by using this strategy such as compact broadband couplers [Nguyen \& Caloz 2007], broadband phase shifters [Eleftheriades \& Balmain, 2005], compact wideband filters[Gil et al., 2007], compact resonant antennas [Lee et al., 2005, 2006; Schüßler et al., 2004; Sanada et al. 2004].

\section{Spiral fractal resonator based compact band-stop filter}

In this section, a compact, low insertion loss, high selective band-stop filter is explained. The filter is composed of two unit cells of electrically small artificial magnetic metamaterials, which have the topological form of fractal spiral resonators in [Palandoken \& Henke, 2009]. The geometry of fractal spiral resonators is formed with the direct connection of two concentric Hilbert fractal curves of different dimensions as in a spiral form to excite the magnetic resonance. The operation principle of band-stop filter is based on the excitation of two electrically coupled fractal spiral resonators through direct connection with the feeding line.

The geometry of one unit cell of periodic artificial magnetic material is described shortly in Section 3.1 along with the reflection/transmission parameters and the resonant field pattern at the magnetic resonance frequency. In Section 3.2, the proposed filter topology is depicted with the geometrical and material parameters. The logical approach of the filter design is explained. In Section 3.3, the transmission and reflection parameters are illustrated to verify the desired filter performance and the operation principle is clarified in the light of simulated current distribution at the stopband frequency.

\subsection{Structural design of fractal spiral resonator}

The geometry of the artificial magnetic material is shown in Fig. 5. Each of the outer and inner rings are the mirrored image of the first order Hilbert fractal to form the ring shape. They are then connected at one end to obtain the spiral form from these two concentric Hilbert fractal curves. The marked inner section is the extension of the inner Hilbert curve so as to increase the resonant length due to the increased inductive and capacitive coupling between the different sections. The substrate material is standard $0.5 \mathrm{~mm}$ thick FR4 with dielectric constant 4.4 and $\tan (\delta)$ 0.02. The metallization is copper. The copper line width and minimum distance between any two lines are $0.2 \mathrm{~mm}$. The other geometrical parameters are $\mathrm{L} 1=2.2 \mathrm{~mm}, \mathrm{~L} 2=0.8 \mathrm{~mm}$ and $\mathrm{L} 3=1 \mathrm{~mm}$. 
The unit cell size is ax $=5 \mathrm{~mm}$, ay $=2 \mathrm{~mm}$, az $=5 \mathrm{~mm}$. Only one side of the substrate is structured with the prescribed fractal geometry while leaving the other side without any metal layer. Because two unit cells of this spiral fractal geometry is excited through the direct connection with the feeding line in the proposed band-stop filter, it is important to verify the magnetic resonance frequency as this frequency is the stopband frequency at which no field transmission is allowed.

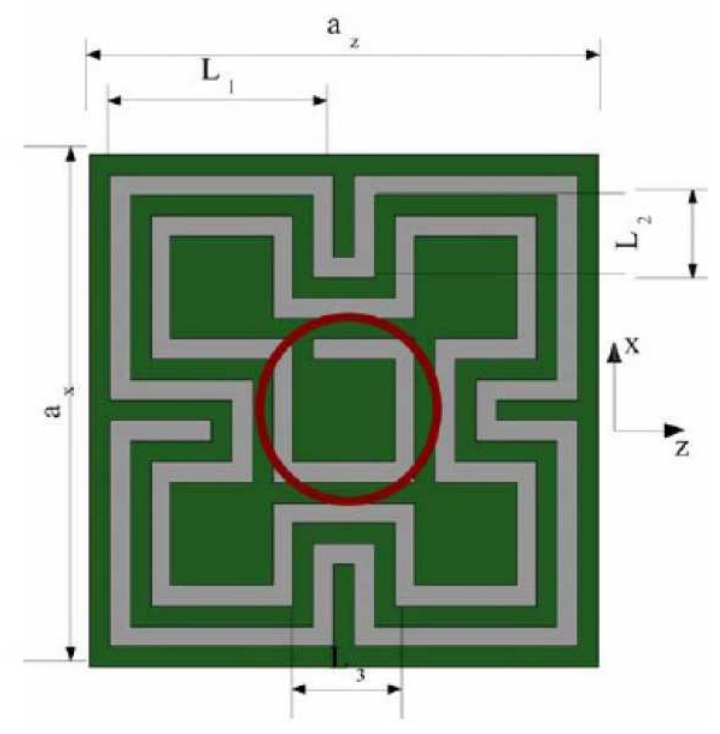

Fig. 5. Fractal spiral resonator geometry

In order to induce the magnetic resonance for the determination of stopband frequency, the structure has to be excited with out-off- plane directed magnetic field. Thus, in the numerical model, the structure is excited by $\mathrm{z}$-direction propagating, $\mathrm{x}$-direction polarized plane wave. Perfect Electric Conductor at two x planes and Perfect Magnetic Conductor at two y planes are assigned as the boundary conditions.

The resonance frequency and surface current distribution at the resonance frequency are numerically calculated with FEM based commercial software HFSS. The simulated Sparameters and surface current distribution are shown in Fig. 6 and Fig. 7, respectively.

Due to the spiralling form of surface current and the resulting out-off-plane directed magnetic field, the magnetic resonance is quite effective. Therefore, this electrically small structure can be regarded as a resonant magnetic dipole at $1.52 \mathrm{GHz}$. The transmission deep through one unit cell-thick artificial material in Fig.6 is effectively due to the depolarization effect of this magnetic dipole for the incoming field. This is the reason why this artificial magnetic material is regarded as a negative permeability material in a certain frequency band and the frequency of transmission deep is regarded as the magnetic resonance frequency of the electrically small structure. This frequency band is the desired band at which the proposed band-stop filter is designed to operate and the incoming field is exponentially attenuated in the propagation direction. 


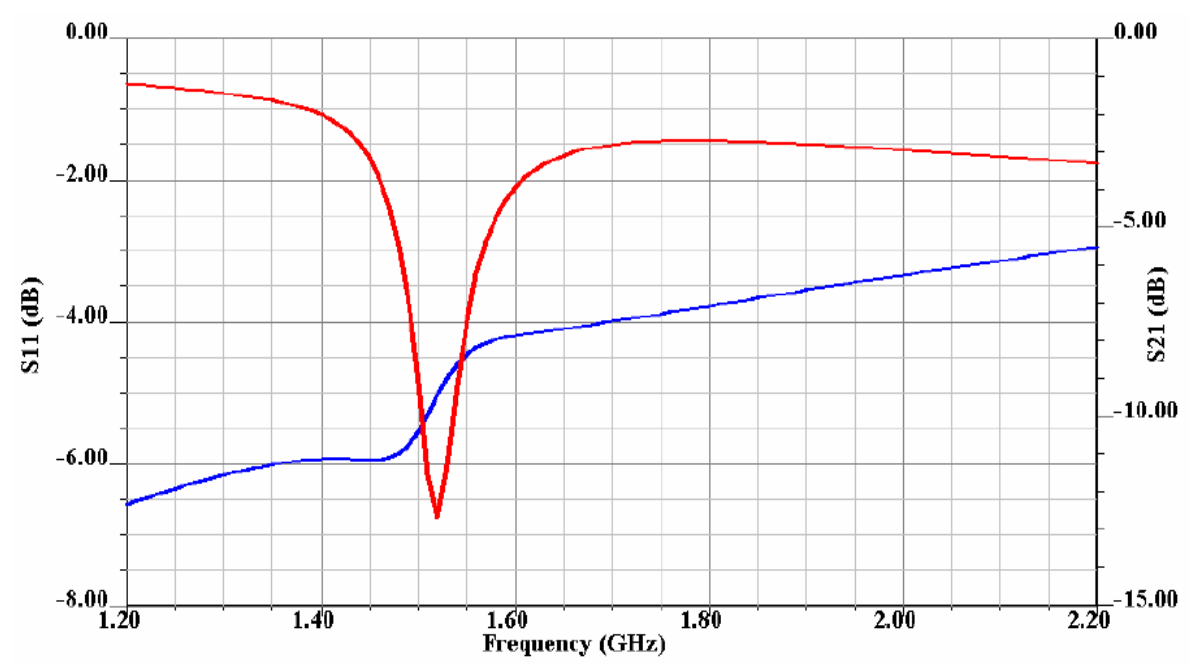

Fig. 6. Transmission (red) and reflection (blue) parameters of fractal spiral resonator

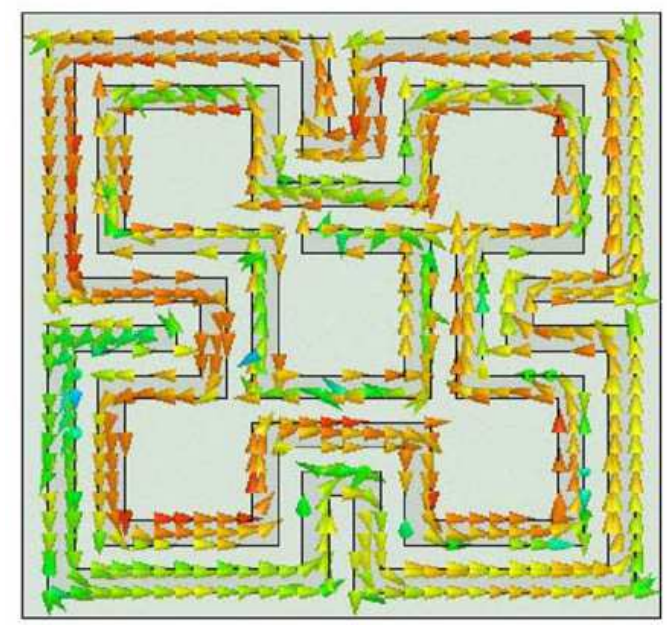

Fig. 7. Surface current distribution of fractal spiral resonator at $1.52 \mathrm{GHz}$

\subsection{Band-stop filter design}

The proposed band-stop filter is shown in Fig. 8. As it could be deduced from the filter topology, two fractal resonators are connected antisymmetrically along the $\mathrm{x}$ axis through the feeding line to have symmetrical return loss. In the filter model, the separation distance between $x$-direction oriented cells is $0.1 \mathrm{~mm}$. Each unit cell is connected directly with the feeding line to increase the field coupling as shown with the red circles in Fig. 8 as a feeding method [Palandoken \& Henke, 2010]. One conventional feeding method is to have resonator shaped slots in the ground plane for high field rejection [Marques et al., 2008]. However, 
this design method is not selected in order to suppress the resulting back radiation due to the slotted ground plane for lower insertion loss. In addition, this type of resonator feeding improves the filter selectivity in comparison to the proximity coupled feeding method. The separation distance between the unit cells and feeding line sections is $0.2 \mathrm{~mm}$. Rather than using high lossy FR4, in the filter model low loss Rogers 4003 material with relative permittivity 3.38 and loss tangent 0.0027 is used. The main reason to use low-loss substrate instead of high-loss FR4 substrate is not only to decrease the insertion loss with low loss substrate but also with low permittivity material to increase the field coupling from the feeding line to the fractal spiral resonators.

The filter width (Wf) and length (Lf) are $10.1 \mathrm{~mm}$ and $5.4 \mathrm{~mm}$, respectively. The width of each metallic line is $0.2 \mathrm{~mm}$ except the width of feeding line, which is $1.1 \mathrm{~mm}$ to excite both resonators effectively and couple each of the resonators with $50 \Omega$ line impedance at both ports. The length of feeding line sections at each port is $5 \mathrm{~mm}$. The total size of band-stop filter is $15.4 \mathrm{~mm}$. The design principle of the proposed filter is to feed the fractal resonators directly through the feeding line with their resonant field distributions. This field distribution is the resonant field excited at the band edge of the bandgap in the Brilliouin diagram, which results into no transmission of the incoming wave because of its standing wave nature. As it could be noticed in Section 3.1, the resonance frequency of fractal spiral resonators is numerically calculated with the electrical coupling between the in-plane oriented cells and magnetic coupling between the out-plane oriented cells in the transverse plane. The feeding method, which is currently exploited in this design, results these fractal spiral resonators to couple electrically as in the numerical model in Section 3.1. As a next step, the return and insertion loss of the band-stop filter is numerically calculated in addition to the resonant surface current distribution.

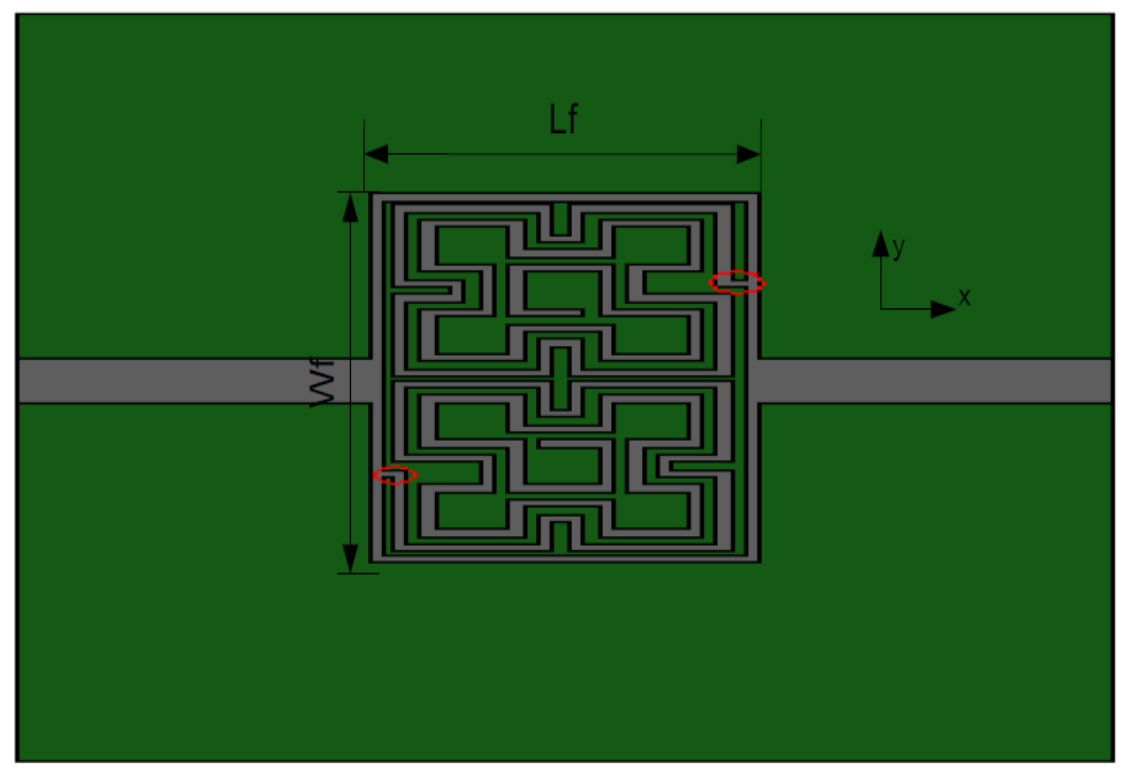

Fig. 8. Fractal spiral resonator based band-stop filter 


\subsection{Numerical results of fractal spiral resonator based band-stop filter}

To validate the design concept, the performance of the band-stop filter is numerically calculated by using FEM based commercial software HFSS. The simulation results of the insertion and return losses are shown in Fig. 9. As shown in Fig. 9, the return loss is larger than $10 \mathrm{~dB}$ in two frequency bands of which is smaller than $0.75 \mathrm{GHz}$ and which is larger than $1.87 \mathrm{GHz}$ in the frequency span of $0.5-2.5 \mathrm{GHz}$. The insertion loss in the passband is better than $1 \mathrm{~dB}$. The frequency rejection level is larger than $20 \mathrm{~dB}$ in the frequency band of $1.36-1.40 \mathrm{GHz}$ and $27 \mathrm{~dB}$ at the center frequency, $1.38 \mathrm{GHz}$. The selectivity of the proposed band-stop filter is quite promising, which is $100 \mathrm{~dB} / \mathrm{GHz}$ frequency selectivity with $3 \mathrm{~dB}$ reference insertion loss.

The physical size of the main filtering section is $\lambda \mathrm{o} / 40.18 \times \lambda_{\mathrm{o}} / 21.48$ at the center frequency, which is quite compact in comparison to the conventional stepped impedance or coupled line filters [Pozar, 2004]. On the other hand, there is no matching network, which is quite advantageous in the filter design to reduce the filter physical size. The total size of the filter even with the transmission line sections at the input and output ports is also compact, which is $\lambda \mathrm{o} / 10.85 \times \lambda_{\mathrm{o}} / 14.46$. The surface current distribution at the center frequency of the stopband, $1.38 \mathrm{GHz}$ is shown in Fig. 10 to verify the design principle.

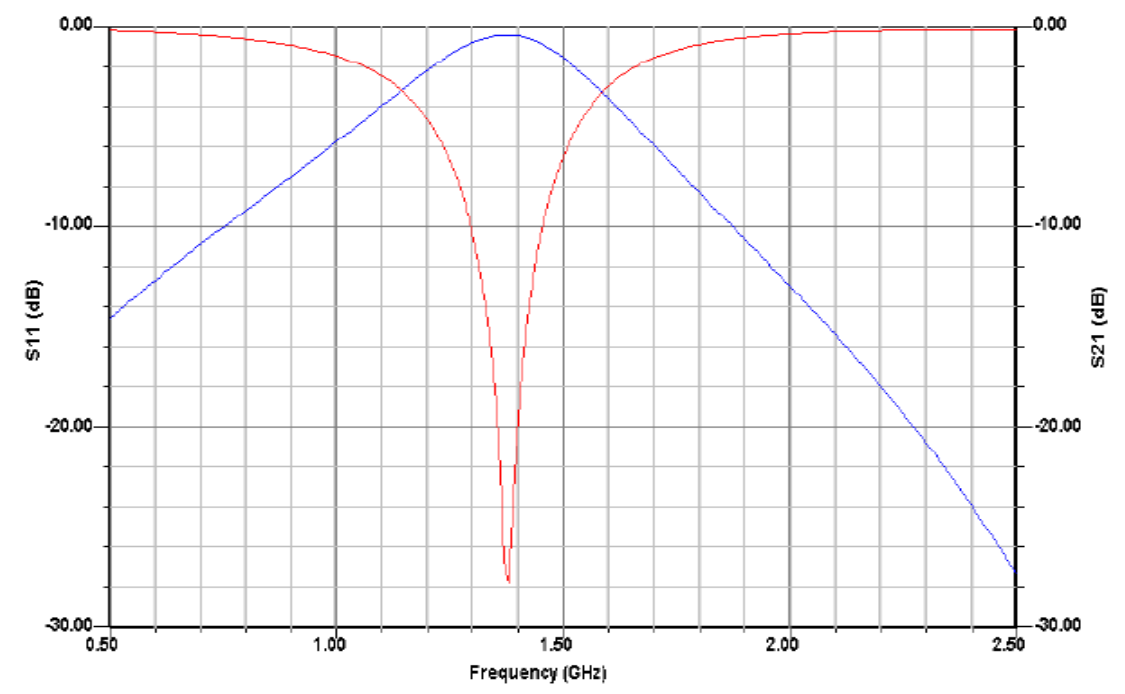

Fig. 9. Reflection(blue) and transmission (red) parameters of band-stop filter

As shown in Fig. 10, two fractal resonators are excited through the direct connection with the feeding line in their resonant current distribution. This type of resonator feeding leads these electrically small cells to have same direction directed magnetic dipole moments, which is actually the same field distribution for two electrically coupled spiral resonators. However, because of the additional electrical length due to the feeding line section for both of the spiral resonators, the center frequency of the stopband is lower than the resonance frequency of the fractal resonators. 


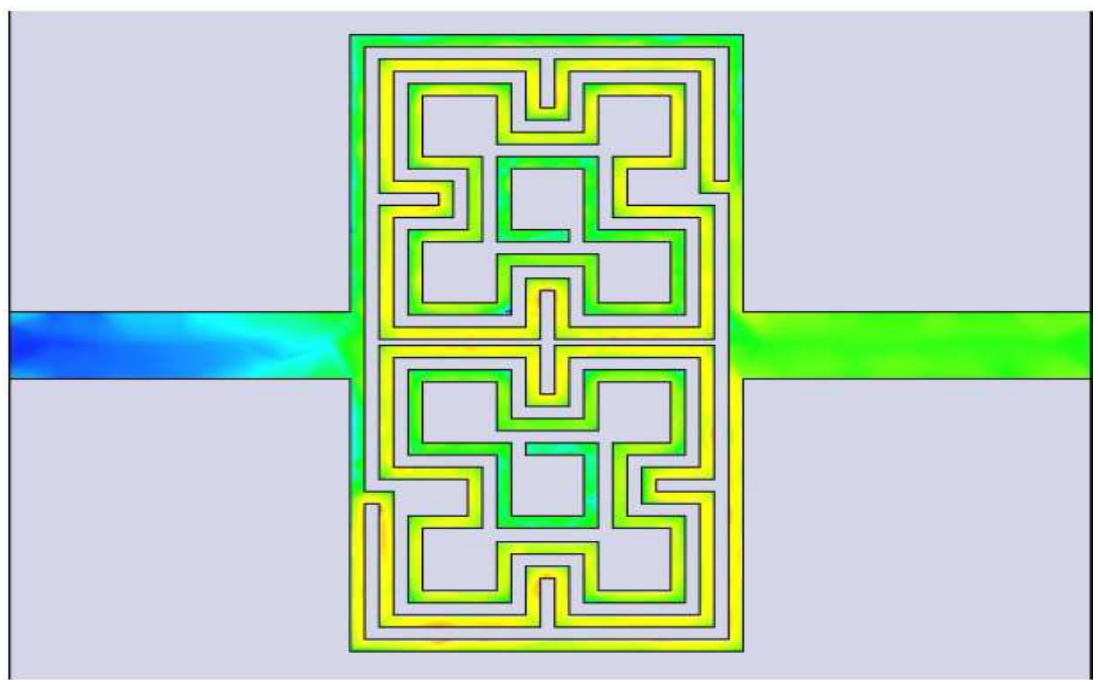

Fig. 10. Surface current distribution of fractal spiral resonator based BSF at $1.38 \mathrm{GHz}$

The operation principle of the proposed filter is thus based on the suppression of the incoming field through the direct coupling of the feeding line to the spiral resonators at the resonant frequency. The resonant field, which is confined at the spiral resonators do not lead the magnetic dipole-like resonators to couple effectively to the electric dipole-like transmission line section at the second port. This reduces the transmission of the incoming field though the input feeding line and increases the reflection of the input signal at the first port.

As a result, in this section, the design of a compact, high selective, low insertion loss band-stop filter with two unit cells of novel magnetic metamaterial geometry is introduced. The physical dimensions of the designed band-stop filter are $\lambda \mathrm{o} / 10.85 \times \lambda_{\mathrm{o}} / 14.46$ with the additional transmission line sections at the center frequency of the stopband . No matching network is required in filter design, which is quite important to reduce the total filter size effectively. The insertion and return losses are numerically calculated and the current distribution at the center frequency of the stopband is illustrated. The proposed filter has satisfactory insertion loss, which is better than $1 \mathrm{~dB}$. The selectivity of the filter is $100 \mathrm{~dB} / \mathrm{GHz}$, which is quite suitable to be used as band-stop filter in modern communication systems.

\section{Thin wire loaded spiral resonator based compact band-pass filter}

In this section, the design of a compact, LHM-based band-pass filter (BPF) is explained. The band-pass filter is composed of two unit cells of electrically small LHMs, which have the same geometry of thin wire loaded spiral resonators in [palandoken et al., 2009]. The LHM geometry is based on the direct connection of thin wire with the spiral resonator in order to enhance the magnetic field coupling inbetween in addition to the increased electrical length for reduced resonance frequency. The operation principle of BPF is based on the excitation of two coupled LHM resonators at two eigenfrequencies through direct connection with the feeding line. 


\subsection{Structural design of thin wire loaded spiral resonator}

LHM behavior relies on the simultaneous excitation of electric and magnetic dipole-like electrically small cells at the resonance frequency. One well-known miniaturization method of such resonators is to increase the field coupling among the individual resonators. This design strategy is chosen for the LHM cell exploited in current BPF design. The LHM geometry is shown in Fig. 11 along with the boundary conditions and excitation sources. The wire strips and spiral resonators (SR) are directly connected with each other on both sides of the substrate. Further, instead of SRRs as in the original proposal of artificial magnetic material, SRs are used, which have half the resonance frequency of SRRs [Baena et al., 2004, 2005 ]. In the design, the geometrical parameters of the front and back side unit cells are the same, except a $0.6 \mathrm{~mm}$ shorter wire strip length on the front side. Different strip wire lengths lead to a smaller resonance frequency and larger bandwidth [palandoken et al., 2009]. The substrate material is nonmagnetic FR4-Epoxy with a relative permittivity of 4.4 and loss tangent of 0.02 .

The frequency dispersive property of FR4 has not been taken into account in the numerical calculation. The validity of the model is illustrated by retrieving the effective constitutive parameters from $S$ parameters and by the opposite direction of group and phase velocity in [palandoken et al., 2009].

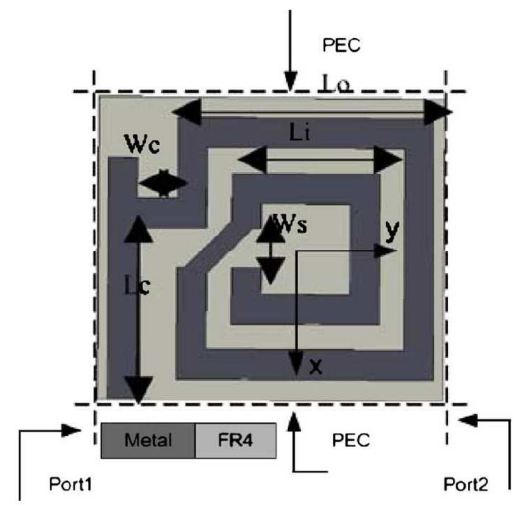

(a)

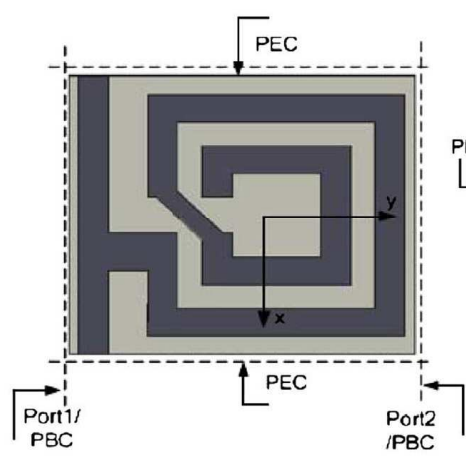

(b)

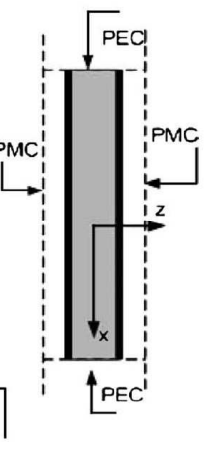

(c)

Fig. 11. LHM unit cell geometry. (a) Front and (b) back side of one LHM unit cell with indicated geometrical parameters in [palandoken et al., 2009].

\subsection{Band-pass filter design}

The band-pass filter is shown in Fig. 12. As it could be implied from the filter topology, two LHM resonators are connected antisymmetrically along the y axis through the feeding line in order to have symmetrical reflection parameter. In the filter model, the separation distance between $\mathrm{x}$-direction oriented cells is $0.2 \mathrm{~mm}$. Each unit cell is connected directly with the feeding line to excite each of LHM resonators effectively and couple the excited LHM resonator electrically and magnetically with the another resonator depending on the mode of excitation in addition to the extended feeding line sections as an effective feeding 
method. LHM resonator shaped slots in the ground plane are not exploited in the current band-pass filter due to the resulting back radiation from the slotted ground plane. The separation distance between one unit cell and feeding line sections in y-direction is $0.8 \mathrm{~mm}$. One LHM resonator is shifted from the another resonator along y-direction with the distance of $0.4 \mathrm{~mm}$ to obtain optimum field intercoupling. Instead of using high lossy FR4, in the filter model low loss Rogers 5880 material with the relative permittivity 2.2 and loss tangent 0.0009 is used. The main reason to use low-loss substrate instead of high-loss FR4 substrate is not only to decrease the insertion loss with low loss substrate but also with low permittivity material to increase the field coupling from the first resonator into the second resonator in addition to the field coupling from the feeding line to each of LHM resonators.

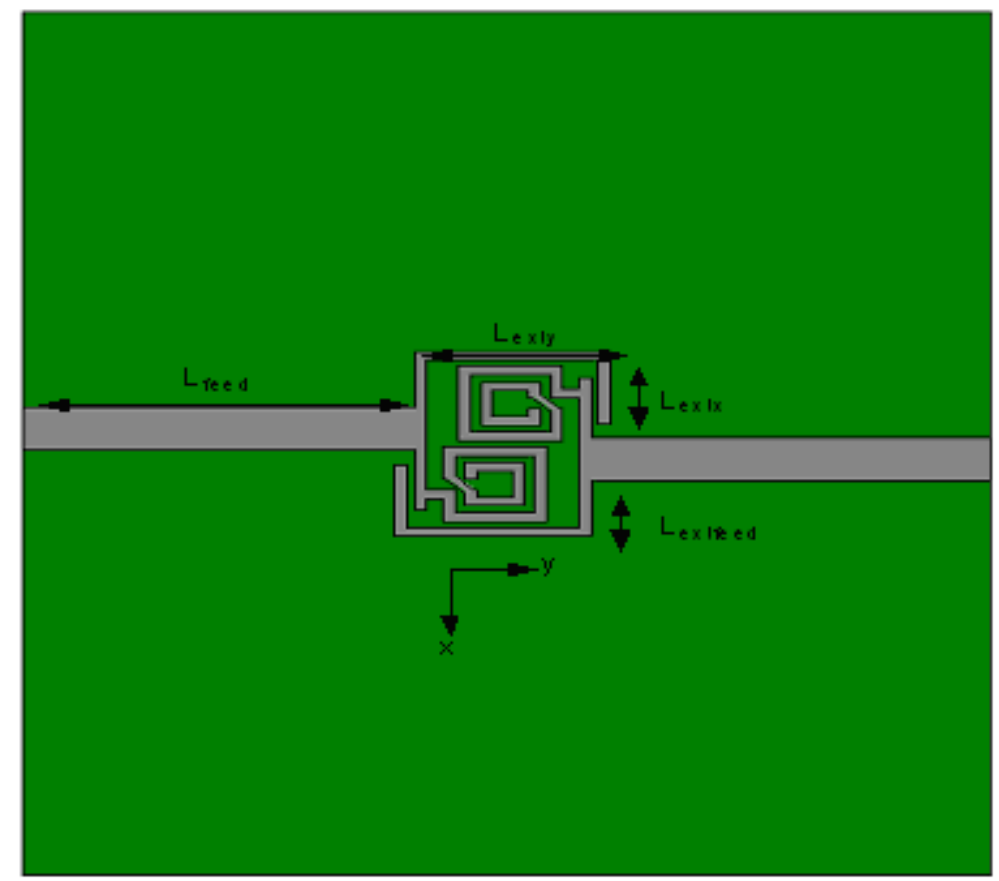

Fig. 12. Thin wire loaded spiral resonator based BPF

The lengths of extended feeding line in $\mathrm{x}-\left(\mathrm{L}_{\text {extx }}\right)$ and y-directions ( $\left.\mathrm{L}_{\text {exty }}\right)$ are $2.5 \mathrm{~mm}$ and $4.9 \mathrm{~mm}$, respectively. The width and length $\left(\mathrm{L}_{\text {feed }}\right)$ of feeding line are $1.5 \mathrm{~mm}$ and $9.8 \mathrm{~mm}$, respectively. The direct connection length of the feeding line, ( $\left.\mathrm{L}_{\text {extfeed }}\right)$ in the extended sections is $1.9 \mathrm{~mm}$. The width and length of Rogers substrate are $30 \mathrm{~mm}$ and $24.2 \mathrm{~mm}$, respectively. The total size of band-pass filter is $6.4 \mathrm{~mm} \times 5.4 \mathrm{~mm}$.

The design principle of the proposed filter is to feed one of the LHM resonators directly through the feeding line with its resonant field distribution. The highly concentrated field distribution results the directly excited first resonator to couple to the second LHM resonator electrically and magnetically depending on the excited 0 and 180 modes. The excited field in the first LHM resonator is enhanced by the magnetic field of the extended 
section of the feeding line. The feeding method, which is currently exploited in this design, results these LHM resonators to couple feeding line in an optimal manner. As a next step, the return and insertion loss of the band-pass filter is numerically calculated in addition to the resonant surface current distribution at two eigenfrequencies.

\subsection{Numerical results of thin wire loaded spiral resonator based band-pass filter}

To validate the filter design concept, the reflection and transmission parameters of bandpass filter are numerically calculated by using FEM based commercial software HFSS. The numerical results are shown in Fig. 13. As deduced from Fig. 13, the return loss is larger than $10 \mathrm{~dB}$ with the insertion loss smaller than $1.3 \mathrm{~dB}$ in the frequency band from $4.49 \mathrm{GHz}$ upto $4.92 \mathrm{GHz}$. The lowest insertion loss is $0.4 \mathrm{~dB}$ at $4.83 \mathrm{GHz}$. The filter selectivities, roll-off factors, are approximately $400 \mathrm{~dB} / \mathrm{GHz}$ and $62 \mathrm{~dB} / \mathrm{GHz}$ at the lower and higher edge of the passband, respectively. The filter response is quite similar to the transfer function of Chebychev filter due to existing passband ripples and steep roll-off from the passband edges to the stop-band frequencies.

The physical size of the filtering section without the feeding line sections is $\lambda_{\mathrm{o}} / 10 \times \lambda_{\mathrm{o}} / 12$ at the center frequency of $4.7 \mathrm{GHz}$. It is quite compact in comparison to the conventional stepped impedance or coupled line filter designs. One important advantage of the current filter design is to have no additional matching network, which reduces the filter physical size significantly. The surface current distributions at two resonance frequencies, $4.52 \mathrm{GHz}$ and $4.83 \mathrm{GHz}$, are shown in Fig. 14 to verify the design principle.

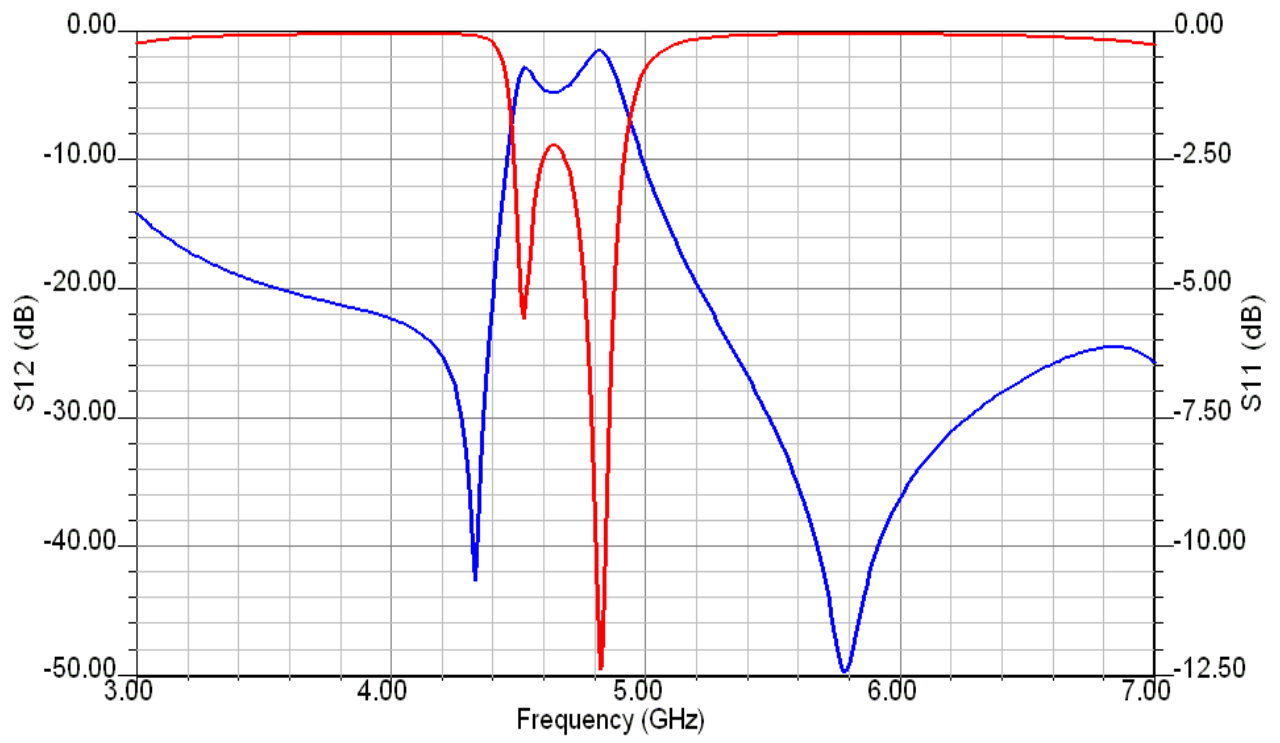

Fig. 13. Reflection(blue) and transmission (red) parameters of band-pass filter 


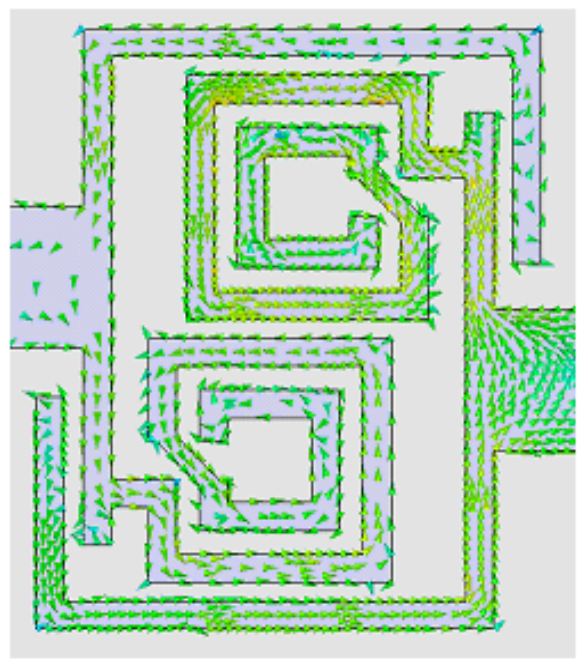

(a)

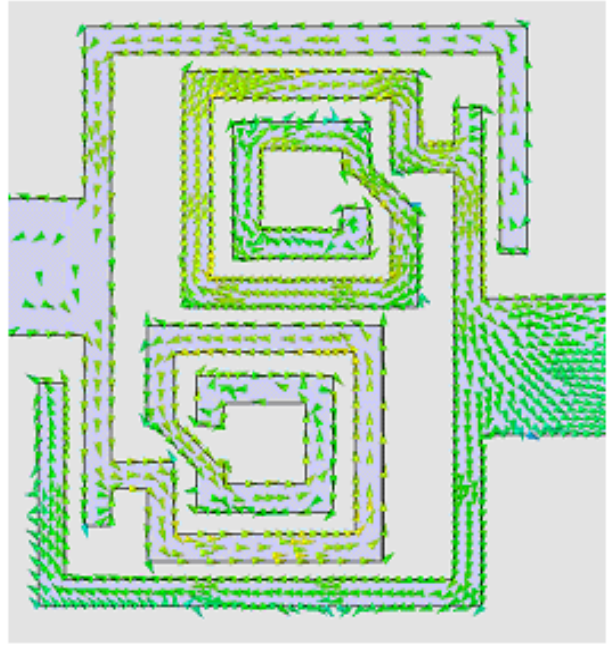

(b)

Fig. 14. Surface current distribution of thin wire loaded spiral resonator based BPF at (a) $4.52 \mathrm{GHz}$ and (b) $4.83 \mathrm{GHz}$

As shown in Fig. 14, one of LHM resonators is directly fed through the feeding line without any metallic connection from the excited resonator to the another LHM resonator. The resonant field distribution of LHM resonator and extended feeding line leads the incoming field to be coupled from the input port to the output port in 0 mode at $4.52 \mathrm{GHz}$ and 180 (л) mode at $4.83 \mathrm{GHz}$. The electric coupling at the lower resonance frequency results both LHM resonators and extended feeding line sections to radiate in comparison to the magnetic coupling at the higher resonance frequency. This is the main reason why the insertion loss is higher at the lower resonance frequency than that at the higher resonance frequency. The transmission principle of incoming field in the passband is based on the excitation of output LHM resonator by the electric and magnetic coupling of the resonant field excited in the directly fed resonator at the lower and higher resonance frequency, respectively.

As a result, in the last section of this chapter, the design of a compact band-pass filter with two unit cells of LHM geometry is explained. The physical dimensions of band-pass filter are $\lambda \mathrm{o} / 10 \times \lambda_{\mathrm{o}} / 12$ at the center frequency of $4.7 \mathrm{GHz}$. There is no need of additional matching network in filter design, which shrinks the total filter size effectively. The insertion and return losses are numerically calculated. The current distributions at two resonance frequencies are illustrated. The proposed filter has satisfactory insertion loss, which is smaller than $1.2 \mathrm{~dB}$ in the frequency band between $4.49 \mathrm{GHz}$ and $4.92 \mathrm{GHz}$ with the bandwidth of $430 \mathrm{MHz}$. The filter selectivities calculated with reference to $3 \mathrm{~dB}$ insertion loss value are $400 \mathrm{~dB} / \mathrm{GHz}$ and $62 \mathrm{~dB} / \mathrm{GHz}$ at the lower and higher edge of the passband, respectively. 


\section{References}

N. G. Alexopoulos, C. A. Kyriazidou, and H. F. Contopanagos, “Effective parameters for metamorphic materials and metamaterials through a resonant inverse scattering approach," IEEE Trans. Microw. Theory Tech., vol. 55, no. 2, pp. 254-267, Feb. 2007

A. Alù and N. Engheta, "Pairing an epsilon-negative slab with a mu-negative slab: Resonance, tunneling and transparency," IEEE Trans. Antennas Propag., vol. 51, no. 10, pp. 2558-2571, Oct. 2003.

A. Alù and N. Engheta, "Guided modes in a waveguide filled with a pair of single-negative(SNG), double-negative (DNG), and/or double-positive (DPS) layers," IEEE Trans. Microw. Theory Tech., vol. 52, no. 1, pp. 199-210, Jan. 2004.

J. Baena, R. Marqués \& F. Medina, “Artificial magnetic metamaterial design by using spiral resonators," Phy. Rev. B, vol. 69, pp. 0144021-0144025, 2004

C.Caloz and T. Itoh, Electromagnetic Metamaterials: Transmission Line Theory and Microwave Applications, Piscataway, NJ: Wiley- IEEE, 2005

X. Chen, T. M. Grzegorczyk, B.-I.Wu, J. Pacheco, Jr., and J. A. Kong, "Robust method to retrieve the constitutive effective parameters of metamaterials," Phys. Rev. Lett. E, vol. 70, pp. 0166081-0166087, 2004.

G. V. Eleftheriades and K. G. Balmain, Negative Refraction Metamaterials: Fundamental Principles and Applications, New York:Wiley Interscience, 2005

N. Engheta, "An idea for thin subwavelength cavity resonators using metamaterials with negative permittivity and permeability," IEEE Antennas Wireless Propag. Lett., vol. 1, no. 1, pp. 10-13, 2002

N. Engheta and R. W. Ziolkowski, Metamaterials Physics and Engineering Explorations, Eds. New York: Wiley/IEEE, 2006.

M. Gil, J. Bonache, J. Garcia-Garcia, J. Martel \& F. Martin, “Composite right/left-handed metamaterial transmission lines based on complementary split-rings resonators and their applications to very wideband and compact filter design," IEEE Trans. Microw. Theory Tech., vol. 55, no. 6, pp. 1296-1304, Jun. 2007.

A. Grbic and G. V. Eleftheriades, "Overcoming the diffraction limit with a planar left- handed transmission lines," Phys. Rev. Lett., vol. 92, no. 11, p. 117 403, Mar. 2004.

A. Grbic and G. V. Eleftheriades, "Experimental verification of backward-wave radiation from a negative refractive index metamaterial," J.Appl. Phys., vol. 92, pp. 59305935, Nov. 2002.

P. Kolinko and D. R. Smith, "Numerical study of electromagnetic waves interacting with negative index materials," Opt. Express, vol. 11, pp. 640-648, Apr. 2003

J. A. Kong, B.-I. Wu, and Y. Zhang, "A unique lateral displacement of a Gaussian beam transmitted through a slab with negative permittivity and permeability," Microwave Opt. Technol. Lett., vol. 33, pp. 136-139, Mar. 2002. 
C.-J. Lee, K. M. K. H. Leong, and T. Itoh, “Composite right/left-handed transmission line based compact resonant antennas for RF module integration," IEEE Trans. Antennas Propag., vol. 54, no. 8, pp. 2283-2291, Aug. 2006.

C.-J. Lee, K. M. K. H. Leong, and T. Itoh, “Design of resonant small antenna using composite right/left handed transmission line," in Antennas Propag. Soc. Int. Symp., 2005, vol. 2B

Ricardo Marqués, Ferran Martín, Mario Sorolla, Metamaterials with negative parameter:theory, design, and microwave applications, John Wiley \& Sons, 2008

H. V. Nguyen and C. Caloz, "Generalized coupled-mode approach of metamaterial coupled line couplers: Coupling theory, phenomenological explanation and experimental demonstration," IEEE Trans. Microw.Theory Tech., vol. 55, no. 5, pp. 1029-1039, May 2007

M. Palandöken, A. Grede \& H. Henke, "Broadband microstrip antenna with LefthandedMetamaterials, "IEEE Trans. Antennas Propag., vol. 57, no.2, pp. 331-338, Feb. 2009

M. Palandöken, H. Henke, “Fractal spiral resonator as magnetic metamaterial, "Applied Electromagnetics Conference, pp. 1-4, 2009

M. Palandöken, H. Henke, "Compact LHM-based band-stop filter", Mediterranean Microwave Symposium, pp. 229-231, Aug. 2010

J. B. Pendry, A. J. Holden, D. J. Robbins \& W. J. Stewart, “Magnetism from conductors and enhanced nonlinear phenomena," IEEE Trans. Microw. Theory Tech., vol. 47, no. 11, pp. 2075-2081, Nov. 1999

J. B. Pendry, A. J. Holden, D. J. Robbins \& W. J. Stewart, "Low-frequency plasmons in thin wire structures," J. Phys., Condens.Matter, vol. 10, pp. 4785-4809, 1998

David Pozar, Microwave Engineering , 2004, Wiley

A. Sanada, M. Kimura, I. Awai, C. Caloz, and T. Itoh, "A planar zeroth- order resonator antenna using a left handed transmission line," in 34th Eur. Microw. Conf., Amsterdam, The Netherlands, 2004, pp. 1341-1344.

M. Schüßler, J. Freese, and R. Jakoby, "Design of compact planar antennas using LHtransmission lines," in Proc. IEEE MTT-S Int. Microw. Symp., 2004, vol. 1, pp. 209212.

D. R. Smith and N. Kroll, "Negative refractive index in left-handed materials," Phys. Rev. Lett., vol. 85, pp. 2933-2936, Oct. 2000.

D. R. Smith, D. C. Vier, N. Kroll, and S. Schultz, "Direct calculation of permeability and permittivity for a left- handed metamaterial," App.Phys. Lett., vol. 77, no. 14, pp. 2246-2248, Oct. 2000

D. R. Smith, D. C. Vier, T. Koschny, and C. M. Soukoulis, "Electromagnetic parameter retrieval from inhomogeneous metamaterials," Phys. Rev. E, vol. 71, pp. 036617103661711, 2005

R.W. Ziolkowski and A. Kipple, "Application of double negative metamaterials to increase the power radiated by electrically small antennas," IEEE Trans. Antennas Propag., vol. 51, no. 10, pp. 2626-2640, Oct. 2003

R. W. Ziolkowski, "Pulsed and CW Gaussian beam interactions with double negative metamaterial slabs," Opt. Express, vol. 11, pp. 662-681, Apr. 2003 
V. G. Veselago, “The electrodynamics of substances with simultaneously negative values of $\varepsilon$ and $\mu$," Sov. Phys. -Usp., vol. 47, pp. 509-514, Jan.-Feb. 1968. 


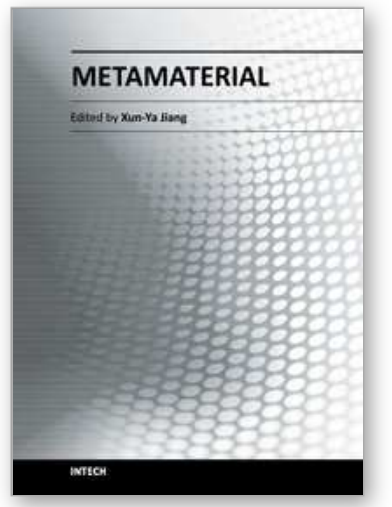

\author{
Metamaterial \\ Edited by Dr. Xun-Ya Jiang
}

ISBN 978-953-51-0591-6

Hard cover, 620 pages

Publisher InTech

Published online 16, May, 2012

Published in print edition May, 2012

In-depth analysis of the theory, properties and description of the most potential technological applications of metamaterials for the realization of novel devices such as subwavelength lenses, invisibility cloaks, dipole and reflector antennas, high frequency telecommunications, new designs of bandpass filters, absorbers and concentrators of EM waves etc. In order to create a new devices it is necessary to know the main electrodynamical characteristics of metamaterial structures on the basis of which the device is supposed to be created. The electromagnetic wave scattering surfaces built with metamaterials are primarily based on the ability of metamaterials to control the surrounded electromagnetic fields by varying their permeability and permittivity characteristics. The book covers some solutions for microwave wavelength scales as well as exploitation of nanoscale EM wavelength such as visible specter using recent advances of nanotechnology, for instance in the field of nanowires, nanopolymers, carbon nanotubes and graphene. Metamaterial is suitable for scholars from extremely large scientific domain and therefore given to engineers, scientists, graduates and other interested professionals from photonics to nanoscience and from material science to antenna engineering as a comprehensive reference on this artificial materials of tomorrow.

\title{
How to reference
}

In order to correctly reference this scholarly work, feel free to copy and paste the following:

Merih Palandoken (2012). Metamaterial-Based Compact Filter Design, Metamaterial, Dr. Xun-Ya Jiang (Ed.), ISBN: 978-953-51-0591-6, InTech, Available from:

http://www.intechopen.com/books/metamaterial/metamaterial-based-compact-filter-design

\section{INTECH}

open science | open minds

\section{InTech Europe}

University Campus STeP Ri Slavka Krautzeka 83/A 51000 Rijeka, Croatia Phone: +385 (51) 770447

Fax: +385 (51) 686166 www.intechopen.com

\section{InTech China}

Unit 405, Office Block, Hotel Equatorial Shanghai No.65, Yan An Road (West), Shanghai, 200040, China 中国上海市延安西路65号上海国际贵都大饭店办公楼405单元 Phone: +86-21-62489820

Fax: $+86-21-62489821$ 
(C) 2012 The Author(s). Licensee IntechOpen. This is an open access article distributed under the terms of the Creative Commons Attribution 3.0 License, which permits unrestricted use, distribution, and reproduction in any medium, provided the original work is properly cited. 